\title{
Applicable Experiments: Collaborative Models for Material Research
}

Before the 19th century, a buildings tectonics was largely unified. Categorical distinctions among so-called "systems" - structure, enclosure, interior and exterior walls, and circulation were unknown. All of that changed with the advent of the steel frame - vertical circulation, environmental systems, clad surfaces, and curtain walls, newly liberated from any structural role, could be internalized, enclosed, encased, and hidden within other concealing floor plates, walls, and ceiling voids.

Throughout 20th century moderism, little effort was made to revive any sense of tectonic unity, despite new technologies and materials. However recent architecture and buildings of the last decade have begun to explore more ambiguous, hybridized, even blurred convergences of structure and surface. While norms of construction, fabrication and assembly still favor isolating building components, architects are now producing buildings whose elements if not reunified - are far from prevailing dogmas of tectonic isolation or structural separation. If we characterize this prevailing separation of structure and skin of the last century as the thinning out among discreet spatial components with separate identities, perhaps the current focus upon mixed or recoupled elements suggests a thickened reunification of structure and skin. What if structure was no longer internalized, but externalized and brought to the outside? What if bearing itself became less localized or isolated and was dispersed across surfaces that were no longer just columns, floor plates, circulation, or façade alone? It is from these initial questions that the author's research emerged - what would a space of merged tectonics look like using steel, the very material responsible for promoting tectonic separation? How would it be done and how would we as architects need to organize ourselves to effectively engage with 21st century technology and manufacturing?

\section{INTRODUCTION}

This article will present strategies for forging collaborative architectural practices between universities and manufacturing industries. It will focus on current architectural research, its explicit impact on manufacturing processes, and the influence of these processes on design outputs. Specifically, the authors will discuss examples of collaboration between faculty and students at the University at Buffalo Department of Architecture, and manufacturing industries initially based in the Buffalo, but more recently, a network of architects,
CHRISTOPHER ROMANO

University at Buffalo

NICHOLAS BRUSCIA

University at Buffalo 


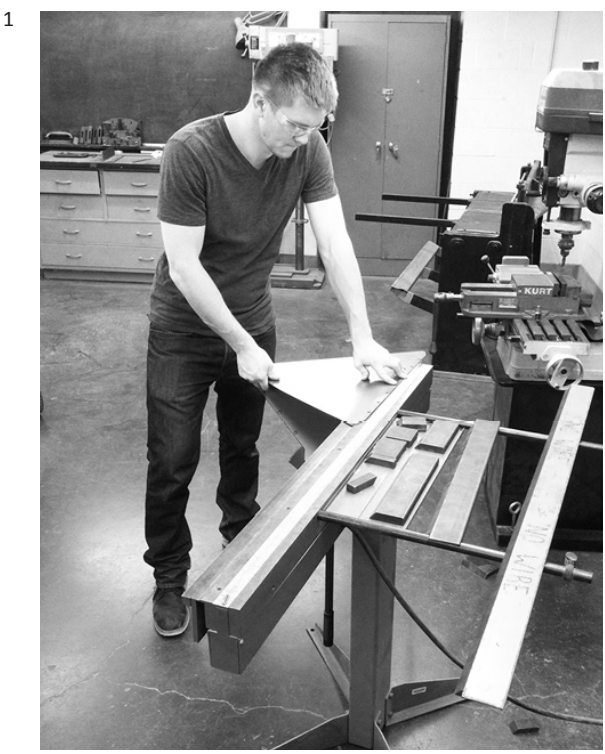

Figure 1: Student assistant working on full-scale prototypes, Materials and Methods Shop, University at Buffalo, Department of Architecture manufacturers, and engineers, spread across the globe. These include: 1) the initiation of a working relationship between the Department of Architecture and the world leader in deeptextured metals manufacturing, Rigidized Metals Corporation, through the development of a student design competition and graduate design seminars, 2) the development of further research by faculty and students on the structural capacities of folding stainless steel and the development of an experimental prototype, project $2 \mathrm{XmT}$, that demonstrates the aesthetic potential of this research, 3) project $3 \times L P$, the winning submission to the international TEX-FAB SKIN competition that coupled the authors and Rigidized Metals with material and fabrication support from A. Zahner Company and engineering support from ARUP, thereby rapidly expanding the reach and momentum of an emerging research practice, and (4) forging new collaborations with manufacturing companies spread more broadly across the globe, specifically the Absolute Joint System (AJ) based in London, England, allowing our team to integrate a wider range of materials and digital technologies into our workflow, while moving our research toward a marketable product.

Using the framework outlined above, this article has four thematic objectives: to describe the procedures by which two faculty developed, designed, and fabricated a prototype in collaboration with an industrial manufacturer, and utilized the described framework in developing a proposal for an intense collaboration; to identify how architects are utilizing digital tools to facilitate collaboration among diverse Architecture, Engineering, and Construction (AEC) teams with the goal of merging design and construction into an integrated workflow; to catalog how new means of interfacing with information and an increased proximity to the production of products/objects through CNC machinery is radically reshaping a renewed material-centric practice in architecture and its related fields; and to assess the capacity of parametric design software and how this method of working/ thinking has allowed architects to streamline drawing-to-production methods.

\section{MODEL 1: FACULTY AS ORGANIZER}

The first collaborative model begins on campus under a loose pedagogical theme to explore architectural applications for a local textured metals manufacturer based in Buffalo, NY, the Rigidized Metals Corporation (RMC). The company is unique for their cold-rolling process of embossing geometric textures into thin gauge metals. The approach was strategically split between two graduate seminars, one being led by Nicholas Bruscia which focused primarily on specular effect through subtle geometric variation (patterns and folds) and the other led by Christopher Romano which focused on self-structuring thin-gauge metal surfaces using similar methods. It was an exploratory phase with students testing a number of preliminary issues that were based on individual interests: unrolling geometric surfaces, folding metal, and mapping a range of specular qualities inherent in the metal. This semesterlong process included a tour of the manufacturing facility, an introduction to the material and manufacturing process by Rick Smith, president of the company, and week-to-week feedback provided by the course instructors. This structure resulted in a series of studentgroups working collaboratively on small physical prototypes using the tools and technology available to them within the university to simulate the effects of rigidized metal and the fabrication workflow of the manufacturer. There were no monetary or logistical risks assumed by the manufacturer, as the large majority of the research was being conducted within the university. At the conclusion of the semester, students presented their work to the president of the company and a small group of administrative staff at RMC.

To begin to unpack the initial benefits of this type of interaction, the manufacturer had a large group of students, near graduation and about to enter the workforce, touring their facilities and learning about their product which alone is an enormous benefit to any manufacturer. In addition, students free from any economic or logistic constraints were able to ask questions, design freely, and introduce contemporary parametric software 
to the manufacturer, which we felt could be of potential use to the manufacturer in the future. As a model that is implemented in the initial phases of a manufacturing relationship, it is useful for the structure to be more traditional so as to allow the academy to engage with imaginative thought experiments based on real-world material contingencies. The advantages for all parties emerge naturally as the relationship moves away from students, faculty, and manufacturer's simply using new information toward the completion of a single self-guided project, to the production of new knowledge whose ultimate goal is real-world applicability.

\section{MODEL 2: FACULTY AS MATERIAL RESEARCHER}

The second collaborative model proposes a faculty-directed research structure that allows the Department of Architecture and local and regional manufacturing to collaborate on the development and full-scale testing of architectural applications for their product line. This includes finding new potential in existing products, the development of new techniques, and the optimization of existing processes through the use of digital tools for both design and fabrication. The research collaboration detailed below is used to explain how this model builds on prior collaborative work completed within two graduate technical methods seminars described in Model 1, while synthesizing these pedagogical approaches into a singular research proposal.

In this model, much of the effort is twofold - material testing a manufacturer's existing product line and attempting to extract the unwritten knowledge that collectively exists amongst the manufacturer's fabrication team. A challenging, yet crucial next step is attempting to document this data in a quantifiable and measurable format that can be used to inform future design decisions. To that end, we conducted extensive testing: photographic documentation of the exterior light reflecting and diffusing qualities of textured metal under a range of weather conditions, strength comparisons of plain stainless vs. textured stainless, 3-point deflection testing of some of the more common patterns to pinpoint which patterns yielded the highest structural performance, and extensive metal folding studies to reveal to the academic team the fabrication limits of both the hydraulic turret punch and hydraulic press brake. This testing yielded a decision-making process that was based on empirical data instead of relying on rule of thumb or repeated cycles of trial and error.

To understand how the research was framed, it is important to have a more detailed understand of rigidizing as a manufacturing process - rolling geometric textures into ordinary sheet-metals to increase the cross-sectional depth of thin-gauges by distributing metal above and below the neutral axis, resulting in a much stiffer material and providing thinner and lighter gauges with increased structural capacity. At the same time, the process gives the material dynamic light diffusing qualities, suppressing oil-canning and resulting in superior optical flatness and uniform aesthetic appearance. To summarize, both specular quality and surface rigidity result from the same geometric conditioning of the metal - a material characteristic that had not previously been studied or exploited. Prior to our collaboration, the exclusive use of this material had been for non-structural façade elements or interior panels backed by substrates, the intention of this research is to develop a self-supporting architectural system that reveals the existing, but underutilized, structural potential of the material while simultaneously exhibiting the specular quality of the texture. Our research culminated in an experimental prototype, project $2 X m T$, which uses a framework of arrayed octahedrons and thin-gauge rigidized metal to generate a selfstructuring skin that exhibits extreme physical and visual lightness. Based on the textural qualities of the metal and the principle of triangulation, specifically through the use of an expanded diagrid, the authors invented an ultra-thin, woven "face-frame" - a space frame

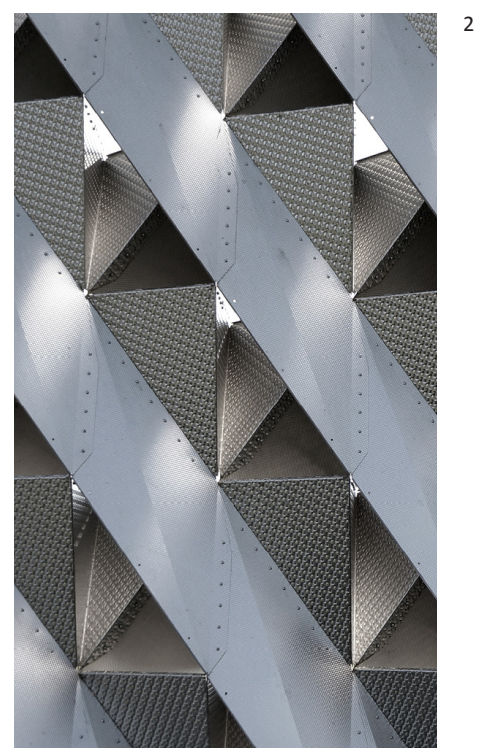

Figure 2: project $2 X m T$ - detail of rear elevation showing expanded/woven diagrid and the combination of metal textures 


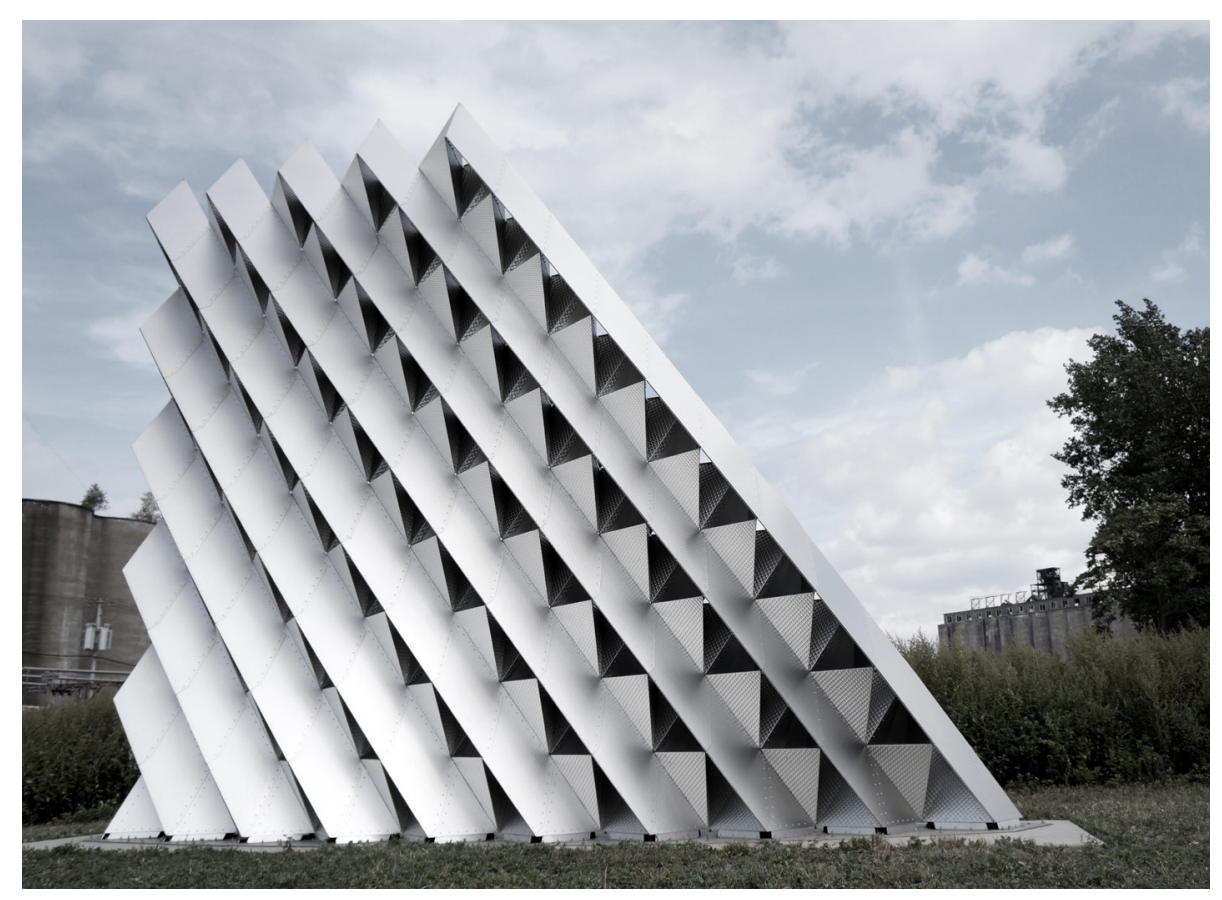

turned into surfaces where instead of connective nodes, overlaps in the surfaces make for a more continuous and more effective connection by better distributing the forces moving through the metal. What is made clear through this work is that the rigidizing process simultaneously creates a visual and structural potency, making large scale thin-gauge assemblages possible. ${ }^{1}$ At a height of $19^{\prime}-6^{\prime \prime}(5.8 \mathrm{~m})$, project $2 \mathrm{XmT}$ is the world's-largest selfstructuring surface, and to our knowledge we can use this framework to scale up infinitely.

As a collaborative approach, project $2 \mathrm{XmT}$ can be compared to the Los Angeles County Museum of Art's (LACMA) original Art and Technology program, which ran from 1967 to 1971. Curator Maurice Tuchman invited artists to be matched with companies working in industry; pairing Tony Smith with the Container Corporation, a manufacturer of paperboard products including folding cartons, paper bags, and fiber cans. Up until that time, most of Smith's sculptures were generated from modular-based paperboard components, typically tetrahedrons or octahedrons, but the component nature of his work became invisible once the work was fabricated in steel at a much larger scale. Working with the Container Corporation, he could replicate his method of working at a monumental scale - resulting in a 2,500 unit cave-like exhibition for the U.S. Pavilion at Expo '70 in Osaka, Japan. ${ }^{2}$ As in our case, the installation achieved a more precise level of clarity when artist and industry collaborate on research.

This model of collaboration, closely aligned with the academic, privately-sponsored research project, requires a great deal of time to frame the research in a manner that is mutually beneficial to all parties. Working agreements are signed that outline the scope of the research, project expectations are agreed upon, and monies exchange hands to execute the research. In working with RMC, it became clear that the project would entail far more oversight from the manufacturer, would require regular meetings with the fabrication team, and that we would be integrated into the monthly production cycle as if we were a paying customer. As a model, it requires financial support, large quantities of raw material, and high demands on machine time and human labor. But, if successful, the research would dramatically increase the visibility and marketability of their product line. In addition to the potential marketing benefits, it was equally as beneficial from a technical perspective. The digital tools we were introducing were not part of the day-to-day workflow 
of the manufacturer, which has since changed as the result of our work and our attempt to demonstrate their relevance in advanced manufacturing. Furthermore, by discussing the project in a parametrically-controlled digital model, architects and fabricators are able to speak the same language and clearly visualize information. This three-dimensional conversation allows the fabricators to work off more accurate base files, reduce mistakes, and thus minimize risk. It also results in better coordination amongst team members and in a faster fabrication schedule. For our team, this digitally-based workflow reinforces our appreciation for mathematics, allowing us to be more creative and explore more complex geometries that were not familiar to the fabricator, thus spending additional time on design that would have otherwise been dedicated to project coordination. More importantly, this collaboration allows us access to cutting-edge machinery and the ability to test ideas at a much larger scale than previously possible, re-centering the material mockup as a crucial and necessary part of the architectural design process.

\section{MODEL 3: FACULTY AS CONDUIT}

The third collaborative model was a two-day specialized workshop which was part of the 2013 International ACADIA Adaptive Architecture Conference. The workshop covered topics ranging from scripting, simulation of complex systems, and digital fabrication with advanced manufacturers. As workshop directors, we were interested in getting students and professionals to interact directly with the fabrication team with the primary goal of getting participants on the factory floor with the people who make things, observing the process of how their drawings are translated to generate $\mathrm{CNC}$ code that can be read by the machinery in the facility. For many participants, this is their first time on a factory floor exploring a type of making that is unfamiliar to many of them: making with very-large machines. At a minimum, we wanted participants to understand how to effectively communicate with fabricators.

Throughout the two-day workshop "Rigidized Metal Forming," we were tasked with consolidating what we had learned in one year into a 48-hour period, taking students through the entire design-to-fabrication process. Participants were constantly moving between analog and parametric modeling, realizing a small, but critical lesson as stated by the French engineer Robert Le Ricolais, "The art of structure is about where to place the holes." Even in a very brief period of time, the opportunity to speak directly with fabricators, touch and feel the metal, and assemble a prototype of their own design changed the way participants thought about material and fabrication. In addition, the manufacturer's affiliation with the ACADIA community gave their product wide exposure both domestically and internationally through the hundreds of student, academic, and professional attendees from around the world. The workshop model is an effective method for closing the gap between the academy and the profession and perhaps more importantly, breaking from the traditional university course structure of meeting once or twice/week for 15 weeks. From our experience, the workshop model is based on brief, but uninterrupted periods of intense learning, and is able to produce similar results in terms of output and quality when compared to typical university coursework, such as described in Model 1.

\section{MODEL 4: FACULTY AS TACTICIAN}

The fourth model of collaboration was a repeat of the latter half of Model 2 (Faculty as Material Scientist), except that it was now a long-distance collaboration amongst many parties involving a commissioning agent serving as the role of client, a number of universities that make up the TEX-FAB Digital Fabrication Alliance, A. Zahner Co. as fabrication sponsor, and ARUP as engineering sponsor. The project needed to be completed in a matter of weeks, not months, thus we saw ourselves in a new role - that of tactician, with a large majority of our time and energy dedicating to managing the relationships 


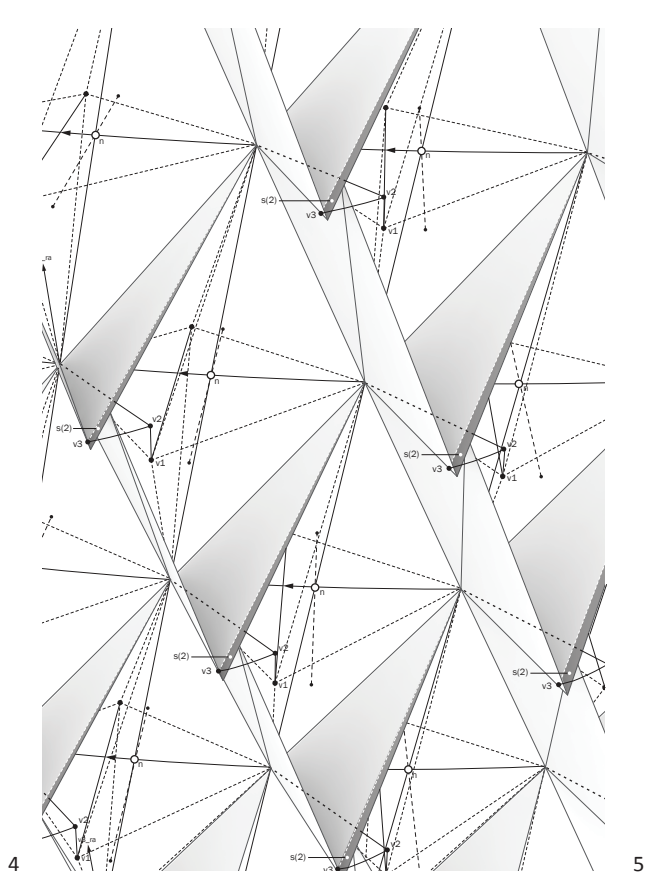

Figure 4: project 3xLP - partial view of drawing showing geometric parameters and variation

Figure 5: project 3xLP - rear elevation view, Tex Fab 5 - SKIN: Digital

Assemblies exhibition, Austin Texas between a greatly expanded team of stakeholders. An added challenge was that this research would have to be conducted remotely with very little face-to-face communication which was vital to the success of previous models.

As part of our TEX-FAB SKIN competition winning entry, project $3 x L P$, we were granted the opportunity to build a second iteration of our SKIN prototype, refining and experimenting with our self-structuring system to introduce visual porosity while maintaining structural stability. Our first assignment was to negotiate bringing Rigidized Metals onboard as both co-material and co-fabrication sponsor. This strategy allowed us to continue to work with the material central to the research, and not knowingly, more than double the funding available to execute the second prototype, thereby increasing the scale and scope of the second prototype. With little time to build physical mock-ups, we opted to digitally simulate the effect of physical forces with the assistance of Maria Mingallon, a structural engineer at ARUP, performing an initial round of FEA analysis on the second prototype, creating a feedback loop between digital model, our first physical prototype, and stress-based FEA analysis. Stated Mingallon, "The results of the digital analysis demonstrated that the origami-like strategy would make the wall strong enough to deal with the typical design loads applied to medium-height buildings." ${ }^{3}$ This feedback provided a level of confidence that we could apply this system at a much larger scale and as a contemporary façade solution.

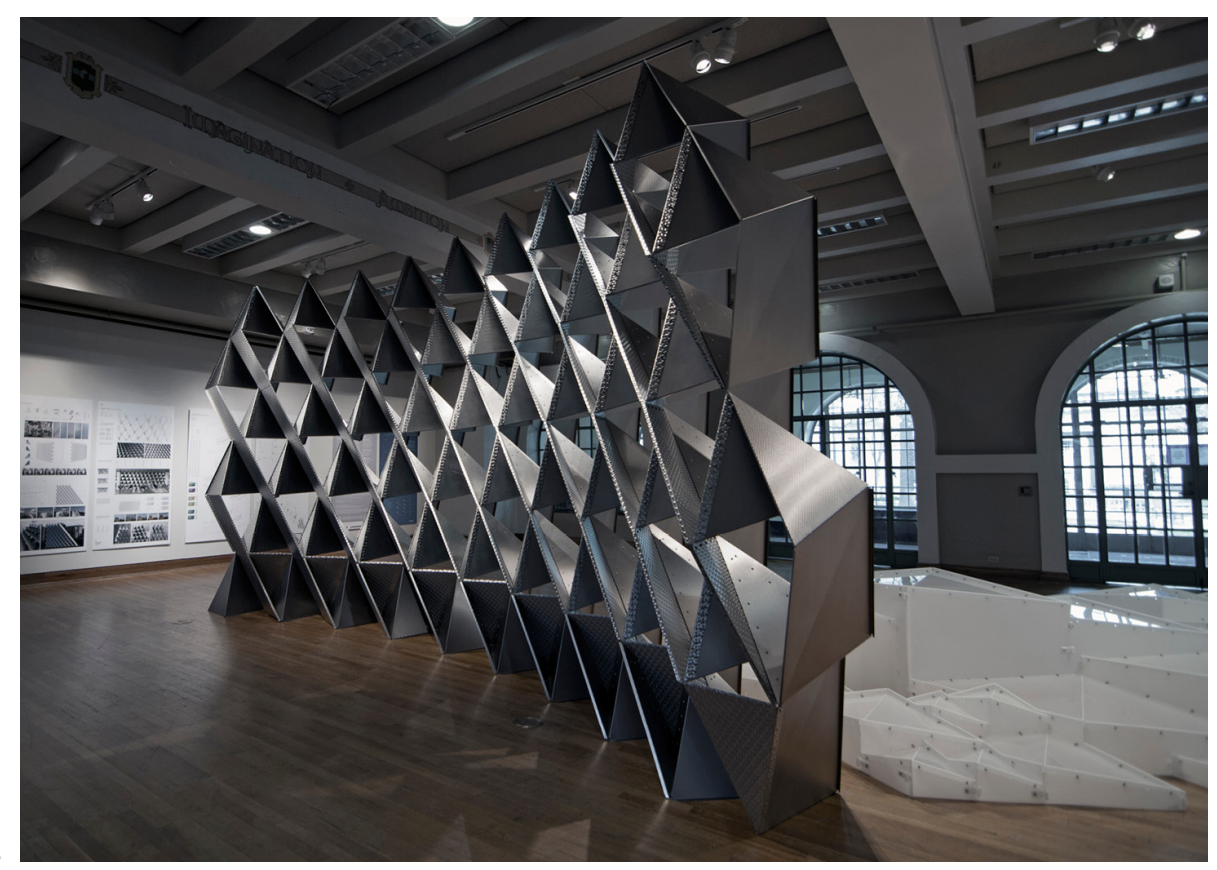

As mentioned above, this collaborative model was about speed, expanding outreach, and relying on external expertise to complete the project. There was little time to meet in person, to design, to prototype, and to make a fabrication error that would alter the production schedule. In doing so, our three-dimensional modeling got tighter, containing more precise data regarding part numbers, geometric families, patterns, gauges, grains, finishes, and assembly sequence. Our need for traditional drawings was dramatically reduced (but not eliminated), resulting in time gained for iteratively testing design solutions and resolving details to achieve tighter fabrication tolerances. We also began to optimize design parameters to find a suitable balance between geometric variation, machine time, and human labor. The competition platform provided a showcase to demonstrate the 
fabrication capacity of two expert manufacturing companies. Repeating the process strengthened and solidified our working relationship with RMC, while allowing us to collaborate with A. Zahner Co., a world leader in metal façade manufacturing. From a marketing perspective, the benefits were measurable as the results were included in various print/online publications and numerous contacts were made within Texas.

\section{MODEL 5: FACULTY AS PROCESS ENGINEER}

The fifth model of collaboration is a joint-venture partnership forged between a public research institution and a privately-held company, latter one focused primarily on the development of large-scale, modular building structures and the former, primarily on the development of innovative building skins whose collaboration attempts to further develop a more marketable product that could be more widely distributed in global architectural contexts. In early conversations, both parties were interested in forming an interdisciplinary partnership that would merge the two threads of research together to form a more holistic system that could deliver architectural solutions for both structure and skin.

The authors teamed up with Bartolomeo Mongiardino and Alessandro Traverso, mechanical engineers based in London, England, and inventors of the Absolute Joint System (AJ), one of two non-welded, round pipe, stainless steel structural systems in the world. More specifically, AJ is a dismountable and reusable space frame system with members connected by means of custom spherical joints. Targeting reusability in lieu of recyclability, the AJ system is a highly durable kit-of-parts for small and large scale space frames that can adapt to a wide-range of spatial configurations to reduce waste and minimize the embodied energy required to create building structures. ${ }^{4}$ Our collaborators recognized that there is an increase in the production of temporal programs that require large expanses of column-free space, such as temporary shelters, storage/transportation facilities, and large stadiums whose intended lifespan may be shorter than traditional buildings. In contrast to the costly maintenance and (often times) infrequent use of these permanent structures, the dismountable AJ system proposes an alternative approach to construction.

In response to the agenda set by B. Mongiardino and A. Traverso, the work was summarized very broadly, working simultaneously towards three goals: 1 ) to increase the feasibility of the AJ System, 2) troubleshoot their existing web-based product offering, and 3) test their structural system against a range of geometries, enclosure systems, and panelization options. Currently, we are focusing on the development of surface optimization and efficient panelization using rigidized metal that can adapt to multiple configurations. Similar to the concept of the AJ system, we are attempting to develop a series of identical panel families that can be applied to formally distinct free-form surfaces. By designing a kit-of-panels, we are attempting to construct a full-scale mock-up that explores reusability in large-scale architecture: a reconfigurable kit-of-parts, structure and skin, that can be mounted and dismounted, packed in a cargo container, shipped across the globe, and reconstructed in a range of configurations.

For the authors, this work has many benefits. It is research that directly engages in the construction industry to develop solutions that find efficiencies in problems that have existed within the discipline for decades. At the same time, this collaboration furthers the research, moving beyond the initial question of self-structuring of thin-gauge, textured stainless steel to addressing questions about enclosure and a higher degree of performance. In addition, it allows the work to move toward a marketable product that could very quickly reach a global audience and do so at a large-scale. For RMC, it is an ideal application for their deep-textured products: lightweight, durable, and highly resistant to visible scratching, making it ideal for structures that are repeatedly assembled and dis-assembled. For the AJ Team, this collaboration gives them a base of operations in the United States, a 
manufacturing partner in RMC, and the ability to test their system on a range of complex geometries prior to entering into the highly competitive material manufacturing market.

\section{MODEL 6: UNIVERSITY AS INCUBATOR}

In this last model of collaboration, our role shifts from faculty-directed research to that of architectural consultant with workflow moving through the manufacturer. In the contemporary AEC industry, there is a reoccurring pattern where clients are looking for the manufacturer to provide in-house expertise to solve technical and logistical issues that arise throughout the design and implementation process. As the research moves from sponsored-research to for-profit commissions and consultations, we have found a usefulness for a young design practice that can move fluidly between a design-assist and a design-led format depending on the scale, scope, and scheduling of an incoming project. When not acting in a traditional architectural role, we operate as an alternative mode of practice, hovering between academia and industry, and thus able to provide a number of alternative benefits: mediating between architects and manufacturing throughout all phases of the design process, teaching sales and marketing teams about emerging technologies in architecture, and focusing on commissioned work that exists somewhere between the scale of installation and architecture. This newfound capacity allows RMC to take on work it would have otherwise turned away, thereby increasing internal capacity and allowing a greater audience access to their product offerings. In this scenario, both university-supported and industry-supported work generates an incubator where young design practices can balance their intellectual curiosity with 75 years of industry expertise.

\section{CONCLUSION}

In conclusion, these are models that we are exploring as alternative modes to traditional architectural practice. The models suggest that these are not idiosyncratic moments/ relationships, but rather, educational, research, and practice models that can be replicable in other locations, with other companies, and sustained for the long-term. Although each of these models are capable of standing-alone, they can be performed in succession as a relationship-building strategy, or they can simultaneously overlap, where one model can serve as a test-bed for the other. Nonetheless, it is through initiating a conversation about computation-tied-to-making that we are able to directly engage in the supply chain, allowing architects and manufacturers to develop a collective-intelligence and a highly collaborative workflow. Through the use of these organizational models, parametrically controlled three-dimensional modeling, and an extreme attention to detail in the manufacturing process, we argue that we are increasing the scope of architecture - taking control of project delivery back into the realm of the architect and reconstituting a high level of craft through intense collaboration with manufacturing. It is through this confluence of interests in both digital technology and contemporary industry that has offered us a way to push forward an alternative trajectory of architectural research and practice.

\section{ACKNOWLEDGEMENTS}

The research has been made possible through the generous sponsorship and enthusiasm of Rick Smith, Chip Skop, Kevin Porteus, Kevin Fuller, Tom Schunk and the expert knowledge of the fabrication team at the Rigidized Metals Corporation. The research agenda has also been supported by Omar Khan and the University at Buffalo Department of Architecture, Association for Computer Aided Design in Architecture (ACADIA), the TEX-FAB Digital Fabrication Alliance: Andrew Vrana, Kevin McClellan, Kory Bieg, and Brad Bell, A. Zahner Company: Bill Zahner, Jim Porter, and Randy Stratman, and Maria Mingallon at ARUP. The efforts of student assistants have played a critical role in the research at all phases: Daniel Fiore, Philip Gusmano, David Heaton, Yibo Jiao, and Daniel Vrana. 